Revista Educación 25(2): 113-124, 2001

\title{
EL JUEGO EN LOS NIÑOS: ENFOQUE TEÓRICO
}

\author{
Maureen Meneses Montero \\ María de los Ángeles Monge Alvarado
}

\begin{abstract}
Resumen: El juego es una actividad tan antigua como el hombre mismo, aunque su concepto, y su forma de practicarlo varía según la cultura de los pueblos. El ser humano lo realiza en forma innata, producto de una experiencia placentera como resultado de un compromiso en particular, es un estímulo valioso mediante el cual el individuo se vuelve más hábil, perspicaz, ligero, diestro, fuerte y sobre todo alegre, asi lo definen Lacayo y Coello (1992), donde también consideran que los niños aprenden a crecer en una forma recreativa.

El docente debe estar consciente de la utilidad del juego en el desarrollo de aprendizajes, su labor se va a ver facilitada en el sentido de tener objetivos de clase, actividades estructuradas no solo de expresión libre sino de desarrollo social, emocional e intelectual.

El artículo pretende dar generalidades de lo que es el juego, su importancia para el educador, teorias del juego y sus propulsores, asi como la clasificación de los mismos. Esto permite al docente formular un concepto teórico que sustente las actividades relacionadas entre el juego y sus objetivos de clase. Convirtiendo o dando asi un sentido lógico a una actividad natural del niño y lo que se espera que él desarrolle integralmente en el aula.
\end{abstract}

\section{Introducción}

Gran parte del contenido y significado del juego infantil está constituido por las actividades lúdicas en las que el niño representa algún papel e imita aquel aspecto más significativo de las actividades adultas y de su contacto con ellas. La evolución del niño y el juego está relacionada con el medio en que se desenvuelva, el cual va a mediar en el desarrollo de las habilidades y destrezas.

El juego resulta de una actividad creativa natural, sin aprendizaje anticipado, que proviene de la vida misma. Tanto para el ser humano como para el animal, el juego es una función necesaria y vital.

La acción de jugar es automotivada de acuerdo con los intereses personales o impulsos expresivos. El juego natural tolera todo el rango de habilidades de movimiento, las normas o reglas son autodefinidas, la actividad es espontánea e individual. Es en parte un reflejo del entorno que rodea al individuo; una actividad necesaria, ya que contribuye al desarrollo integral del infante.

Diversos autores, como los que se mencionan a continuación, emiten sus criterios acerca del juego.

Díaz (1993) lo caracteriza como una actividad pura, donde no existe interés alguno; simplemente el jugar es espontáneo, es algo que nace y se exterioriza. Es placentero; hace que la persona se sienta bien. 
Para Flinchum (1988) el juego abastece al niño de libertad para liberar la energía que tiene reprimida, fomenta las habilidades interpersonales y le ayuda a encontrar un lugar en el mundo social.

Jugando, el niño aprende a establecer relaciones sociales con otras personas, se plantea y resuelve problemas propios de la edad.

A pesar de la necesidad que tiene el niño de jugar y de los efectos benéficos que posee el juego, los adultos lo hacen a un lado y no le dan el lugar que merece entre sus actividades porque no brinda ningún provecho económico y tangible.

Para otros, representa una pérdida de tiempo y no creen en la función que ejerce en el niño por lo que los obligan a realizar actividades no propias para su edad, menospreciando los frutos que reciben a través de sus experiencias.

De acuerdo con Brower (1988) el juego no es un lujo, sino una necesidad para todo niño en desarrollo. Según Hetzer (1992) es tal vez la mejor base para una etapa adulta sana, exitosa y plena.

Zapata (1990) afirma que el juego no exige esfuerzo, pero algunos pueden requerir más energía de la necesaria para realizar sus acciones cotidianas.

El juego y las acciones que este conlleva son la base para la educación integral, ya que para su ejecución se requiere de la interacción y de la actitud social. Por otra parte, además de los objetivos afectivos y sociales ya nombrados, también están los cognoscitivos y motores porque solo mediante el dominio de habilidades sociales, cognoscitivas, motrices y afectivas es posible lograr la capacidad de jugar. (Sutton-Smith, 1978).

La actividad del juego se manifiesta en movimientos de diversas clases. Unos aparentan carecer de finalidad, otros están muy adaptados a un objeto (el acto de succión del niño). Hay movimientos que parecieran responder a estímulos exteriores y otros parecen ser la expresión de estados de bienestar o desagrado. También hay otros movimientos llamados "impulsivos", que se caracterizan por la falta de coordinación y de estímulos exteriores que los provoquen.

Conforme el niño crece, los movimientos espontáneos disminuyen y aparecen otros coordinados. Este no se encuentra adaptado todavía a los estímulos ambientales. El niño no puede dominar sus impulsos porque tiene una verdadera necesidad de movimiento. Se agita, corre, salta, brinca. Esto es un signo de inestabilidad, otra característica de la naturaleza juvenil.

El ambiente es desconocido por el niño, poco a poco lo va percibiendo por medio de sus sentidos y empieza a actuar dentro de él, confrontándose con los objetos, los individuos y los animales, por lo que aparecen nuevas formas de juegos.

El juego se caracteriza también como una actividad placentera, ya que consiste en movimientos libres, sin dirección fija ni obstáculo cualquiera.

\section{El papel del juego en el jardín de infantes}

Zapata (1990) acota que el juego es "un elemento primordial en la educación escolar". Los niños aprenden más mientras juegan, por lo que esta actividad debe convertirse en el eje central del programa.

La educación por medio del movimiento hace uso del juego ya que proporciona al niño grandes beneficios, entre los que se puede citar la contribución al desarrollo del potencial cognitivo, la percepción, la activación de la memoria y el arte del lenguaje.

Flinchun (1988) menciona una investigación en la que se reportó que entre el nacimiento hasta los 8 años aproximadamente, el $80 \%$ del aprendizaje individual ya ha ocurrido, y dado que en este tiempo el niño lo que ha hecho ha sido jugar entonces se debe reflexionar sobre el aporte que tiene el juego en el desarrollo cognoscitivo.

Bruner refuerza esta teoría y expone que también contribuye al proceso memorístico (Bequer, 1993). 
Por medio del juego, el niño progresivamente aprende a compartir, a desarrollar conceptos de cooperación y de trabajo común; también aprende a protegerse a sí mismo y defender sus derechos.

El niño corre, salta, trepa, persigue. Estas actividades lo divierten y fortifican sus músculos; por eso, también cuando se arrastra, se estira, alcanza objetos, patea y explora con el cuerpo, aprende a usarlo y a ubicarlo correctamente en el espacio.

Una de las razones por las cuales los niños deben jugar es para contribuir a su desarrollo físico. Sin darse cuenta, ejecutan un movimiento muchas veces hasta que lo dominan. Con esta actitud el niño reafirma y repite un movimiento sin cansarse hasta que este sea perfecto, sólo por el gusto de realizarlo bien.

El juego, además de contribuir en su desarrollo físico, también favorece su desarrollo cultural y emocional. Para el niño con actitudes y conductas inadecuadas, tales como el mal manejo de la frustración, desesperación o rabia, el juego es una salida para liberar esos sentimientos.

Todo lugar es bueno para jugar y hay juegos para todo lugar. Sin embargo, debe haber un espacio especial, privado y respetado por los adultos, decorado según los intereses e inquietudes del niño. Además conforme crezca, ese espacio debe ser más vasto, para que logre jugar sin peligro y sin temor alguno, al permitírsele hacer uso de sus movimientos, imaginación, fantasías y materiales.

La escuela también debe ofrecerle al educando la posibilidad de participar en juegos deportivos organizados y de carácter competitivo. Para ello, organizará campeonatos internos, invitará a otras instituciones y aceptará las que se le hagan para que los estudiantes tengan la oportunidad de asistir.

\section{El rol del educador en el juego}

El educador es un guía y su orientación se da en forma indirecta al crear oportunidades, brindar el tiempo y espacio necesario, proporcionar material y, principalmente, formas de juego de acuerdo con la edad de los educandos.

Al seleccionar el juego el educador debe tomar en cuenta que las experiencias por realizar sean positivas. Debe ser hábil y tener iniciativa y comprensión para entender y resolver favorablemente las situaciones que se le presentan. Si el individuo no resuelve un reto o problema después de varios intentos, es conveniente que el educador le sugiera que se devuelva al anterior de manera que pueda guiarlo para manejar los sentimientos de frustración.

En el juego no competitivo todo está determinado por el grupo o grupos mediante actos comunicativos con todas sus implicaciones pedagógicas como lo son: el tiempo, la hora, el lugar, las medidas del área, hasta la forma de jugarlo.

En el juego competitivo, además de los educadores-entrenadores, hay una tercera persona involucrada llamada: juez o árbitro. Este tiene la función de aplicar el reglamento del deporte, pero su tarea más importante consiste en educar al niño dentro del terreno de juego, ya sea explicando una regla o haciendo un llamado de atención ante la conducta presentada.

El educador cuando hace uso del juego desea que se dé el aprendizaje social, es decir que los alumnos tengan la oportunidad de obtener experiencias sociales y emocionales mientras juegan; por ejemplo: enfado, alegría, agresión, conflicto y otras. Para esto Giebenhain (1982) recomienda que se dé el diálogo, lo cual permitiría la asimilación cognoscitiva de vivencias y así llegar a la conciencia. Por ejemplo, cuando un alumno es golpeado por un contrario, inmediatamente debe haber un pequeño diálogo entre ellos, ya que si no sucede, la agresión se verá fortalecida y nacerán deseos de venganza, algo que no debe de darse, ni siquiera en el deporte. Cuando se habla sobre lo sucedido se contribuye a que estos dos alumnos jueguen limpio.

Este autor recomienda que para reforzar el aprendizaje social, los alumnos deben aprender a resolver sus propios conflictos y a 
poner sus propias reglas. El papel del educador será el de definir el marco metodológico en el que han de moverse sus pupilos e intervenir cuando rompen los principios pedagógicos.

El intercambiar roles, profesor-alumno, alumno-profesor, brindará al educando una vivencia diferente y aprenderá a ver las cosas desde el punto de vista del profesor. La forma para llevar esta vivencia estará a cargo de la creatividad que tenga el docente. La comunicación, una vez más, es la base para lograr una participación masiva del estudiantado en las lecciones; por ello, hay que alimentarla y posibilitarla organizadamente.

Se recomienda a los educadores una serie de principios pedagógicos para la enseñanza de los juegos:

1. Debe conocer muy bien el juego antes de presentarlo a los educandos, tener listo el material por utilizar y delimitar el terreno de juego.

2. Debe motivar a los alumnos antes y después del juego.

3. Debe explicar claramente y en forma sencilla el juego antes de dirigirlo. Además debe exigir la atención de la clase, para lo cual los alumnos deben estar en un lugar donde todos puedan escuchar. Debe dar la oportunidad de que realicen preguntas para un mejor entendimiento.

4. Después de explicar el juego, se demuestra con un pequeño grupo de alumnos o por el profesor.

5. Si no fue lo suficientemente claro, detenga el juego y corrija el error.

6. Si hay un marcador, deje que los jugadores lo vean, y al final mencione al ganador.

7. Antes de iniciar un juego debe haber enseñado sus fundamentos, para así desarrollar las habilidades y destrezas de los educandos.

8. Cuando el grupo está listo, puede implementar variaciones del juego.

9. Si el juego ya se está volviendo monótono debe cambiarse o terminarse, lo que evitará que los niños se cansen o se aburran.

10. El educador debe involucrarse en el juego, mostrando interés en él.

11. Antes de presentar un juego se debe pensar en que todos los alumnos van a participar, y cuando se está practicando si alguno de ellos no lo está haciendo hay que averiguar por qué no lo hace.

12. Cuando se enseña el juego hay que mostrar también sus dificultades y sus peligros.

13. Para mantener el interés del juego y evitar problemas es recomendable que los equipos sean homogéneos y equilibrados en fuerza y habilidad.

14. Se debe tratar que los jugadores que pierden no salgan del todo del juego.

15. Si el grupo es muy grande, se puede subdividir promoviendo así una mayor participación de todo el grupo.

\section{Teóricos del juego y la educación preescolar}

Existen diversos autores que elaboran sus propias teorías sobre el juego, las cuáles varían y se fundamentan de acuerdo con las diferentes formas de jugar y con la formación profesional del investigador

Todos han contribuido a una explicación y ejecución del juego infantil como una expresión natural; una necesidad filogenética del ser humano y un aspecto que influye en el proceso educativo de los niños, sobre todo en la etapa preescolar.

En resumen, algunas de las propuestas teóricas son las siguientes:

- Groos (1896), estudió el juego en los animales y posteriormente en los seres humanos; esto le permitió conocer las características innatas en las especies.

- Claparéde (1900) continúa el estudio de Groos y define la teoría del preejercicio: ejercicios de tendencias instintivas que 
posteriormente encontrarían en la vida adulta, su aplicación concreta en el trabajo, en el deporte o en la vida cotidiana.

El juego, explica, es un ejercicio preparatorio para la vida y tiene como objeto el libre desarrollo de los instintos heredados todavía sin formar; resulta un agente natural educativo. (Zapata, 1990).

- Wallon: considera que el juego se confunde bastante bien con la actividad entera del niño, mientras esta siga siendo espontánea y no reciba sus objetivos de las disciplinas educativas. Por tanto, la escuela debe buscar en el juego infantil un medio y no condicionarlo a finalidades educativas cerradas.

- $\quad$ Froebel (1782-1852), pedagogo alemán, inicia el movimiento de educación preescolar sistemática; de él surgen los centros preescolares como necesidad social y familiar, método natural y activo ya que toma en cuenta la naturaleza infantil y su espontaneidad.

En los jardines infantiles o "Kindergarten", las plantas son los niños y están bajo el estímulo y cuidado de las "jardineras" o maestras.

En el "Kindergarten" el niño desarrolla su individualidad naturalmente gracias a la actividad espontánea, pero se debe procurar el desenvolvimiento social, por medio de un ambiente de colaboración adecuado.

Construyó dones o regalos para el logro de sus objetivos:

1. Una pelota de tela con otras 6 pelotas menores que llevan colores del arcoiris.

2. Una esfera o bola, un cubo, un dado y un cilindro.

3. Un cubo desarmable en 8 más pequeños.

4. Otro cubo que se descompone en 8 tablitas planas.

5. Otro cilindro en 27 dados pequeños entre los que se encuentra alguno diagonal.

6. Un cubo desarmable en 27 tablitas más cantos (trabajo manual).
- María Montessori, (1870-1952) se basa en la teoría para el desarrollo y la liberación de los niños. Considera que el niño necesita mucho cariño pero que está dotado de una inmensa potencialidad latente, el niño es inquieto y en continua transformación corporal.

Los principios que fundamentan esta teoría en relación con el niño son:

Libertad, actividad, vitalidad, individualidad.

La escuela debe brindar al niño un ambiente apropiado en el que pueda actuar con total libertad y pueda encontrar el material y los juguetes didácticos que respondan a su profunda necesidad de moverse, actuar y realizar ejercicios.

El método Montessori concibe la educación como una "auto educación": porque es donde el niño realiza ejercicios de la vida práctica; no hay intervención directa del educador, en razón de que el niño debe hacer su trabajo por sí mismo.

El método se apoya en el asocianismo y por medio del material adecuado se inicia la educación de los sentidos. Entre el material didáctico propuesto se encuentran:

- $\quad$ Encastrables: material para ejercitar la educación y comparación de las cosas.

- Material para ejercitar sentido del tacto (texturas y pesos).

- $\quad$ Ejercicios para el sentido aromático.

- Planos encastrables de figuras geométricas.

- Cuerpos geométricos para el desarrollo del sentido estereognóstico.

- Cajas sonoras.

Otros materiales básicos son el barro, la arena, el agua, los cuentos, el papel blanco, el material para colorear y cualquier material artístico.

- Decroly (1871-1932), trabajó para adecuar las leyes del desarrollo infantil a la

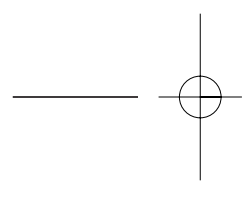


enseñanza con el fin de atender las necesidades de los niños de manera integral.

Los principios fundamentales de su método son:

1. Principio de la globalización

2. Principio del interés

Basado en estos principios contribuye, por medio de la iniciación, a la actividad intelectual y motriz, a los juegos educativos. Adopta la siguiente clasificación:

a. Juegos que se refieren al desarrollo de las preocupaciones sensoriales y la aptitud motriz.

b. Juegos de iniciación matemática.

c. Juegos que se refieren a la noción del tiempo.

d. Juegos de iniciación a la lectura.

e. Juegos de gramática y comprensión del lenguaje.

- $\quad$ Celestín Freinet (1896-1966) propone la teoría de "la educación por el trabajo". Parte de la búsqueda práctica de la educación popular interesante, eficiente y humana sobre todo, en la cual el trabajo se constituye en eje y motor de su desarrollo.

Se basa en 30 principios que deben operar en toda situación educativa, denominados invariables pedagógicos.

Algunos de los principios que se deben considerar son: que se aprende por actividad específica, esto es, se aprende a leer y escribir leyendo y escribiendo; a dibujar se aprende dibujando, etc.; es decir, por medio de la libre exploración y la experimentación el niño aprende y conforma su inteligencia y sus conocimientos.

\section{Teorías del juego}

El estudio de la naturaleza humana y sus diferentes conductas, a través de muchos años ha involucrado una serie de pensadores, enfocados principalmente a explicar el juego y su influencia en el ser humano.

Para Torbert y Schnieder (1986) el juego es "la llave que abre muchas puertas". Erickson y Piaget manifiestan que el juego es "un agitado proceso de la vida del niño"; y White lo resumió como "una diversión, pero también como un serio negocio. Durante esas horas el niño estructura firmemente su aptitud en las relaciones con el ambiente". Todos tratan de resumir conductas del juego y el ser humano y viceversa.

Díaz (1993) resume varias de ellas y las agrupa en lo que llama dos vertientes: $1^{\circ}$. ¿Por qué el niño juega?, llamada teoría eficiente o causal y $2^{\circ}$. ¿Para qué juega el niño?, llamada teoría de causa final o teleológica.

En la primera se encuentran las teorías del descanso y distracción, energía superflua, el atavismo o recapitulación y la catártica.

En las teorías de causa final están: la del ejercicio preparatorio o la de la práctica del instinto, de la derivación por ficción y la psicoanalítica.

Los educadores físicos, maestros y maestras, al iniciar las sesiones de juegos, deben basarse en teorías pedagógicas que les permitan adecuar las actividades y hacer un buen uso de la actividad natural del niño.

\section{Teoría de la energía excedente}

En el siglo XIX, el filósofo inglés Herbet Spencer consideró que el juego se daba por la necesidad de liberar la energía corporal que se tenía en exceso (Kraus, 1990).

Spencer basó su criterio en el de Friedrich von Schiller, quien afirmaba que cuando los animales satisfacían sus necesidades básicas; liberaban la energía excedente por medio de una serie de juegos placenteros e inofensivos (Kraus, 1990).

Spencer vio en el juego de los niños una limitación de las actividades adultas, en las que intervenían "instintos predadores". Él conceptualizó el juego como la base para una serie de actividades humanas (Kraus, 1990).

Es un punto de vista que se orienta principalmente al sector infantil. Se dice que existe 
una superabundancia de energías nerviosas y musculares propias de la fisiología del cuerpo de los niños en edades preescolares y escolares por lo que el profesor de Educación Física deberá balancear las actividades en proporción con las energías que demuestran los alumnos. A mayor energía, más duradera la actividad; o sea hay un cúmulo de energía que no se utiliza en materias teóricas y que se puede usar en Educación Física o en actividades de movimiento (Vargas, 1995).

El pensamiento de Spencer era que para los niños que comen y descansan bien y que no necesitan consumir sus energías para poder sobrevivir, el juego se convierte en un escape para su excedente de energía. (Newmann y Newmann 1983).

\section{Teoría recreativa, de esparcimiento y recuperación}

El alemán Moritz Lazarus (1883) afirmaba que el propósito del juego es conservar o restaurar la energía cuando se está cansado. Él hacía la diferencia entre la energía física y la energía mental. Cuando el cerebro está cansado, es necesario un cambio de actividad hacia el ejercicio físico, esto restaurará la energía nerviosa (Kraus, 1990).

Esta teoría está más enfocada a los adultos, quienes son los más necesitados de recreación para reponerse para el trabajo posterior (Kraus, 1990).

En el juego se utilizan grandes porciones del sistema neuromuscular no agotadas para recuperar el equilibrio, y las energías perdidas. Este equilibrio se produce en el organismo por medio del juego y los deportes (Vargas, 1995).

Newman y Newman (1983) comentan que la frecuencia con que el juego se da entre los niños se debe a la enorme necesidad de esparcimiento que tienen dada la intensidad de energía que utilizan para aprender tantos nuevos conocimientos $\mathrm{y}$ tantas habilidades.

\section{Teoría de la práctica del instinto (ejercicio preparatorio para la vida futura)}

En 1896, Karl Gross realiza una publicación acerca del juego animal y en 1899 acerca del juego de humano (Kraus, 1990).

Para Gross, el juego ayudaba a los animales a sobrevivir, pues por medio de él, aprendían las destrezas necesarias para la vida adulta. Mientras más adaptable e inteligente era una especie, más necesitaba de protección durante la infancia y la niñez para el aprendizaje de las destrezas. Un ejemplo de estas especies es la humana, en la que se practican roles sociales en la niñez, algunos de los cuales son por sexo, como "jugar de casita" (Kraus, 1990).

Gross veía el juego como un instinto sencillo y generalizado. Él hacía la diferencia entre juego y trabajo, pero aceptaba que el trabajo puede incluir elementos de juego (Kraus, 1990).

La actividad lúdica traducida en la actividad del juego presenta las formas más diversas de acuerdo con los intereses biológicos de una edad determinada. El animal y el hombre juegan no por ser jóvenes, sino porque sienten la necesidad de hacerlo (Vargas, 1995).

El niño nace con instintos y habilidades imperfectos que posteriormente se perfeccionan con el juego (Vargas, 1995).

Esta teoría solo se refiere al niño porque: 1- el juego es necesario para el desarrollo físico e intelectual, 2- el aprendizaje de los animales se reduce en el tiempo porque sus instintos son más desarrollados al nacer que los del niño. El niño deberá jugar más en sus diferentes manifestaciones (Vargas, 1995) y lograr así un desarrollo óptimo en las conductas que manifieste.

\section{Teoría de la recapitulación (teoría atávica)}

El psicólogo y pedagogo estadounidence Stanley Hall (1906) creía que los niños 
eran el eslabón en la cadena de la evolución entre los animales y los hombres, estudiando la historia de la raza humana en los juegos de la niñez y la adolescencia (Krauss, 1990).

Es llamada atávica porque proviene del término biológico atavismo que es la tendencia en los seres vivos a la reaparición de caracteres propios de sus antepasados remotos (Vargas, 1995).

Por medio del juego, los niños eran enseñados a revivir la vida de sus ancestros relacionándose en actividades que fueron vitales para la especie, tales como pescar, andar en canoas, cazar y acampar (Krauss, 1990).

Hall demostró que los juegos de los niños reflejan los diferentes periodos de la historia de la humanidad: la etapa "animal", la "salvaje", la "nómada" y la de "tribu" (Krauss, 1990).

El profesor de Educación Física, afirma Vargas (1995), relaciona al individuo con la naturaleza; aprende actividades atávicas (ir a las montañas, valles, lagos, excursiones, pesca, alpinismo, natación, atletismo) que se realizan de generación en generación y no pierden vigencia.

\section{Teoria de la catarsis}

En esta teoría se cree que el juego sirve como una válvula de escape para las emociones reprimidas (Krauss, 1990).

Carr, un psicólogo estadounidense, establece que la catarsis es el "drenaje" de la energía que tiene posibilidades antisociales (Krauss, 1990).

Otro psicólogo, Patrick, establece que las emociones fuertes, como el miedo o la cólera, causan una serie de cambios internos en el organismo que lo exponen a respuestas agotadoras, las cuales conducen al individuo a situaciones amenazantes. Por lo tanto, el juego actuaría como un catalizador de esa energía y ayudaría al cuerpo a recuperar su estado de equilibrio (Krauss, 1990).

Al igual que la Teoría de la Excedente, considera que el juego activo libera la energía excedente y provee un canal socialmente aceptable para la agresión y las emociones hostiles (Krauss, 1990).

Esta teoría es poco aceptada actualmente, aunque se acepta más en las sociedades preindustriales. (Krauss, 1990).

\section{Teoría de la auto expresión}

Elmer Mitchell y Bernard Mason, educadores físicos, consideraban al juego como el resultado de la necesidad de autoexpresión. El juego estaba influenciado por factores psicológicos, anatómicos, la condición física y deseos de la humanidad, tales como el de una nueva experiencia, la participación en empresas grupales, seguridad, respuesta y reconocimiento de otros, y estética (Krauss, 1990).

Vargas (1995) cita a, Clarapede, el cual establece que: 1- la estructura anatómica y fisiológica del hombre lo capacitan para correr, saltar y trepar 2- el uso de estos movimientos provoca la perfección de la función, 3- las características estructurales del organismo influyen en la elección de las actividades del juego, 4- la aptitud física del hombre determina sus preferencias hacia algunas actividades del juego, 5- las inclinaciones psicológicas del hombre lo llevan a ejecutar ciertas actividades del juego.

Por experiencia, el hombre adquiere hábitos de juego. La experiencia se adquiere como actitudes en forma de respuestas de conducta hacia determinados patrones sociales y culturales. Para el hombre identificado con tradiciones culturales, sus hábitos se perfeccionan hasta el grado de que las formas de juegos de un grupo social tienden a constituir también sus hábitos. El individuo toma las costumbres del medio que lo rodea (Vargas, 1995).

\section{Teoria del juego como estimulante de crecimiento}

Vargas (1995) caracteriza a la teoría dentro de un sentido biológico. En 1902, Problst y Cark promulgaron la Ley Biológica de que la función hace al órgano.

A medida que el hombre desarrolla actividad física por medio del juego prepara su 
organismo y lo habilita para obtener mayor y mejor rendimiento.

\section{Teoría del entretenimiento}

Vargas (1995) comenta que esta teoría considera al juego como un mero pasatiempo o diversión, que no tiene mayor significado en la vida. Es una forma de perder el tiempo.

Para algunos profesores de Educación Física, esta teoría tiene valor específico y necesario en caso de reunión festiva, con fines de esparcimiento y regocijo. Entretiene y divierte a la gente (Vargas, 1995).

\section{Teoría del juego como ejercicio complementario}

Vargas, (1995) menciona a H. Carl el cual pensaba que: 1- el juego conserva y renueva los conocimientos, habilidades y destrezas del niño, joven o alumno, y 2- el juego crea nuevos hábitos y perfecciona los ya existentes hasta automatizarlos.

En la educación física, el juego viene a ser un ultra ejercicio: un ejercicio elevado a un grado superior de habilidad y destreza. El educador físico tiende a perfeccionar los hábitos y costumbres del individuo (Vargas, 1995).

\section{Teoría de crecimiento y mejoramiento}

Newman y Newman (1983) hacen mención a Appleton (1910), el cual consideró el juego como un modo de aumentar las capacidades del niño. Lo definía como el tiempo donde los niños descubren y ensaya sus capacidades. Consideraban que el juego dirige al niño hacia una actitud más madura y efectiva.

\section{Teoría de reestructuración cognoscitiva}

Piaget (1951) parte de que el juego es una forma de asimilación. Desde la infancia y a través de la etapa del pensamiento operacional concreto, el niño usa el juego para adaptar los hechos de la realidad a esquemas que ya tiene.

Newman y Newman (1983) comentan que cuando los niños experimentan cosas nuevas, juegan con ellas para encontrar los distintos caminos de cómo el objeto o la situación nueva se asemejan a conceptos ya conocidos.

Además, acotan que Piaget considera el juego como un fenómeno que decrece en importancia en la medida en que el niño adquiere las capacidades intelectuales que le permiten entender la realidad de manera más exacta.

\section{¿Por qué juega el niño?}

El niño juega porque la actividad lúdica le permite ir estructurando y evolucionando en su personalidad. El carácter competitivo, participativo, comunicativo y agonista va adaptándose a los rasgos que rigen esta personalidad. El niño siente el deseo de ejercer un control y dominio total sobre los demás de establecer una comunicación y relación con los que lo rodean por medio de su propio cuerpo y de crear una fantasía liberadora; y encuentra la posibilidad de realizar estos deseos en la actividad lúdica, lo que la define como auténtica expresión del mundo del niño.

El niño actúa en forma positiva el aspecto social al compartir; en el afectivo ya que se conoce más a sí mismo y a los demás y en el cognoscitivo pues desarrolla su intelecto y destrezas.

Hay distintos tipos de juegos para las diferentes edades. Por ejemplo, el juego funcional que es más que todo de manipulación y exploración hasta los seis meses de edad; de uno a dos años el juego de autoafirmación donde el niño conquista una mayor habilidad motora que le va a dar confianza en sus propios medios, autonomía e iniciativa.

El juego de dos a cuatro años se le denomina simbólico. Aquí predominan los juegos de construcción y destrucción. Se comien- 
za a dar la representación por medio de la imitación y simulación de experiencias agradables pasadas, todavía aquí el juego no es compartido, aunque sí necesita el apoyo de algo o alguien.

Por último, el juego pre-social que va de los cuatro a seis años. El niño busca compañeros para sus actividades, pero no es una actividad social ya que el niño ve a sus amigos como si se tratara de juguetes, lo que se denomina juego asociativo.

A esta edad aún no son capaces de organizar un juego, se limitan a asociarse y representar cada uno un papel de forma personal dentro de esa comunidad.

Al usar el juego como un recurso educativo, se deben de tener en cuenta las siguientes consideraciones:

\section{El juego como recreo}

"Recrearse" es formarse de nuevo (etimológicamente hablando). Si esto fuera realmente así el juego sería una reparación, una restitución o regeneración de las fuerzas físicas y mentales fatigadas por el trabajo. Pero esto no es del todo verdadero.

El hombre busca el porqué de la actividad lúdica. El hombre aplica al juego cierta clase de actividad y las fuerzas gastadas en otras formas pueden descansar y repararse.

En el niño este hecho varía, ya que el juego parece ser continuo.

\section{El juego como excedente de energía}

Todo órgano que ha estado en reposo por un tiempo está en situación análoga a la de una pila cargada de electricidad, en tensión creciente que se descarga con la acción.

Spencer ve en el juego una parodia de la vida seria en la que se emplea la energía solamente.

\section{La función biológica del juego}

El psicólogo Karl Gross afirma que el juego es un problema biológico. (Krauss, 1990).
Los niños y los animales jóvenes realizan movimientos coordinados. Tienen juegos como la caza o la lucha, que son formas importantes y fundamentales.

Estos juegos son preejercicios. Son ensayos, determinados tanteos, experimentaciones, es cierto grado de las actividades serias que deberán llenar la vida. Su objetivo es prepararlos para la existencia y estar listos para la lucha. Gross (Krauss,1990) dice que el niño no entra en la vida completamente listo. Tiene un periodo de desarrollo y de crecimiento, esto comprende un tiempo de aprendizaje, un periodo de formación y adquisición de aptitudes y conocimientos.

El juego tiene por objeto desarrollar instintos útiles en la vida, el desarrollo de los órganos y los instintos se debe a la selección natural.

\section{Clasificación del juego según las cualidades que desarrolla}

Díaz (1993) realiza una clasificación de los juegos según las cualidades que desarrollan, como por ejemplo:

1. JUEGOS SENSORIALES: desarrollan los diferentes sentidos del ser humano. Se caracterizan por ser pasivos y por promover un predominio de uno o más sentidos en especial.

2. JUEGOS MOTRICES: buscan la madurez de los movimientos en el niño.

3. JUEGOS DE DESARROLLO ANATÓMICO: estimulan el desarrollo muscular y articular del niño.

4. JUEGOS ORGANIZADOS: refuerzan el canal social y el emocional. Pueden tener implícita la enseñanza.

5. JUEGOS PREDEPORTIVOS: incluyen todos los juegos que tienen como función el desarrollo de las destrezas específicas de los diferentes deportes.

6. JUEGOS DEPORTIVOS: su objetivo es desarrollar los fundamentos y la reglamentación de un deporte, como también la competencia y el ganar o perder. 
Bequer, González y Plous (1993) presentan otra clasificación de los juegos con base en distintos criterios como: edad, grado escolar, intensidad del movimiento, forma de participación, ubicación, característica y tipo.

Así, por ejemplo, por edad y escolaridad se tiene la siguiente clasificación:

0 - 5 años: Educación Inicial,

6 - 9 años: Primer ciclo,

10 - 11 años: Segundo ciclo,

12 - 15 años: Tercer ciclo,

16 - 18 años: Cuarto ciclo,

19 en adelante: Quinto ciclo y nivel universitario.

Según la intensidad del movimiento, se dan los juegos móviles (variedad de movimiento), inmóviles (predominio del trabajo mental y psíquico) y los transitorios (combinación de las dos características anteriores).

Por la ubicación, dependiendo de donde se realicen, son interiores o exteriores.

Por la forma de ubicación son individuales o colectivos.

Por su característica o tipo pueden ser: dramatizados, miméticos, sensoriales, persecución, libres, con cantos, creativos, co-educacionales, rítmicos, tradicionales, pre-deportivos, de relevo, constructivos-destructivos.

\section{Conclusión}

- $\quad$ El juego es una actividad innata en los niños y es reconocida por los autores como un elemento esencial en su desarrollo integral.

- La evolución en la actividad lúdica del niño: juego funcional, juego de autoafirmación, juego simbólico, juego presocial etc., permite al niño estructurar su personalidad.

- $\quad$ El juego ofrece al participante una coyuntura para aplicar comportamientos nuevos a la vida cotidiana.

- $\quad$ El maestro y la maestra deben involucrarse en la actividad del juego, esto va a permitirle estrategias didácticas bien orientadas hacia la consecución de los objetivos propuestos.

- Se debe seleccionar una teoría del juego por parte del educador para fundamentar su marco filosófico.

- $\quad$ Retomando, todos los teóricos del juego han contribuido a una explicación y ejecución del juego infantil, como una expresión natural, una necesidad filogenética del ser humano y un aspecto que influye en el proceso educativo de los niños sobre todo en la etapa preescolar.

- Las iniciativas del juego infantil deben respetarse y tratar de explotar esas premisas dentro de los procesos de enseñanza aprendizaje.

- Los principios que comandan la enseñanza de los juegos no deben obviarse en el momento de su puesta en práctica.

- $\quad$ Es importante tomar en cuenta las clasificaciones de los juegos, ya que cada uno puede desarrollar y fomentar cualidades específicas en el participante.

- La variedad de juegos existentes son innumerables por lo que se aconseja modificarlos continuamente para lograr la integralidad que se busca.

Para finalizar, los juegos ofrecen experiencias de gran valor y versatilidad para el desarrollo integral, por lo que deben ser tomados en cuenta dentro de todo programa de educación formal y no formal.

\section{Referencias bibliográficas}

Bequer, G. et al. Juegos de Movimiento. Unidad Impresora José Huelga. Cuba: INDER. 1993.

Diaz, A. et al. Desarrollo Curricular para la Formación de Maestros Especialistas en Educación Física. España: Editorial Gymnos. 1993.

Flinchun, B. "Early Childhood Movement Programs. Preparing Teachers for 
Tomorrow". Journal physical Education, Recreation and Dance. 59(7) 62-67. 1988

Gebauer, K. Proyecto de Juego. Madrid: Editorial Interduc, S.A. 1979.

Giebenhain, H. Sportsspiel und Sozialisation. Schorndorf, Deutschland. 1982.

Hagstrom y Morril. Juegos con Niños. España: Ediciones CEAC. 1981.

Hetzer, H. El juego y los juguetes. Argentina: Editorial Kapeluz. 1992.

Krauss, R. Recreation and Leisure in Modern Society. HarperCollins Publishers ( $4^{\mathrm{a}}$ edición). 1990.

Lacayo, M. y Coello, L. Educación Física, Deporte y Recreación al Alcance de Todos. Honduras: Talleres de NICOP. 1992.

November, J. Experiencias de Juegos con Preescolares. Madrid: Ediciones Morata, S.A. 1983
Newman, B. y Newman, P. Desarrollo del Niño. México: Editorial Limusa. 1983.

Salazar, C.G. Teorías del Juego. Escuela de Educación Física y Deportes, Universidad de Costa Rica. 1995. (Folleto mimeografiado).

Sutton-Smith, B. Die dialektik des spiels. Schondorf, Deutschland. 1978.

Torbert y Schnieder. "Positive Multicultural Interaction". Journal Physical Education, Recreation and Dance. 57(7) 40-44. 1986.

Vargas Z, O. Resumen del capítulo "El principio de la educación del niño". Álbum Curso de Capacitación de Educación Física. Escuela de Educación Física y Deportes, Universidad de Costa Rica. 1995.

Zapata, O. El Aprendizaje por el Juego en la Etapa Maternal y Pre-Escolar. México: Editorial Pax. 1990. 